Instability, Chaos and Predictability in Celestial Mechanics and Stellar Dynamics
Copyright $\odot 1992$ by Nova Science Publishers, Inc. All rights of reproduction in any form reserved. ISBN 1-56072-054-9

\title{
STOCHASTIC INSTABILITY IN GRAVITATING MEDIUM
}

\author{
Irina K. Rozgacheva \\ Sternberg State Astro Inst., 13 Univer. Prospect, \\ 119899 Moscow V-234, Russia
}

\section{ABSTRACT}

Our Universe has probably a space-time with a humpy metric. The space-time has no ndegenerate (unequal) geodesics. The Universe is weakly inhomogeneous. The nonstationary inhomogeneities (humpies) of gravitational field change the redshift of any astronomical object - change brightness (Sachs-Wolfe's effect), and change trajectories of photons. The latter effect is considered here. It is found that a theorem on the null geodesics are instable in the Universe with positive density contrasts. Photons must lose an information of their initial directions of propagation. So such radiation as the Microwave Background Radiation must seem uniform in the Universe.

STOCHASTIC INSTABIL ITY IN GRAVITATING MED IUM

Dynamical systems which have the configuration space with humpies metrics are considered in this note. For example, it is a space to consist of a plane and asymmetric humps.Any dynamical system has probably a humpy configuration space. Below we have analysed the following theorem:

Theorem 1: There is a humpy space $H$. The countable set of charts covers the space $H$. The metric of a chart is $g_{i k(\ell)}$ ' $i, k=0,1,2,3, \ell=1,2, \ldots, N$, and the frame 4 -vectors are $\left(e_{i}^{(a)}\right)(\ell), a=0,1,2,3$ is a number of a frame vector, and

$$
\begin{aligned}
g_{i k(l)=\left(e_{i(a)} e_{k}^{(a)}\right)(l)=\left(e_{i(0)} e_{k}^{(0)}\right.}+e_{i(1)} e_{k}^{(1)}+e_{i(2)} e_{k}^{(2)} \\
\left.+e_{i(3)} e_{k}^{(3)}\right)(l)
\end{aligned}
$$


A condition of dominant energy $R_{i k} p^{i} P^{k} \geq 0$ is realized.

The space $\mathrm{H}$ will be described by $\mathrm{N}$-measured space of 4vectors $\left(y_{i(a)}\right)_{(l)}=g_{i k}\left(e_{(a)}^{i}\right)(l)$ on a local chart $g_{i k}$. Every vector $y_{i(\ell)}$ corresponds to a vector $e_{i(\ell)}$, which is carried parallely itself from $x_{j(l)}$ to $x_{j}$ on a chart $g_{i k}$.

The isotropic geodesics of a space $\left\{\mathrm{y}_{i(l)}\right\}$ are instable if among the vectors $y_{i(l)}$ are not parallel.

Using Theorem 1 we may reformulate the well-known theorem by Kolmogorov, Arnold and Moser to continual systems: the continual system has a stable motion if its configuration space is enough isotropic.

The chart $g_{i k(\ell)}$ of gravitational system has its coordinate time. The $\mathrm{N}-$ measured space $\mathrm{H}$ proves to enable to introduce only one arrow of time $t$ on all space $H$. The increase of the time depends on an increase of Kolmogorov-Sinai's entropy of a manifold $\left\{y_{i(l)}\right\}$ in one-to -one manner.

As an astrophysical application one can consider the trajectories of photons in an expanding and weakly inhomogeneous Universe. The Universe has probably a space-time with a bumpy metric. The null geodesics or the trajectories of photons are unstable in the Universe with positive density contrasts. Photons must lose an information of their initial directions of propagations. So such radiation as the Microwave Background Radiation must seem uniform in the humpy Universe.

1. Let us consider a humpy space $H$ with points $x_{j}$. A hump lies near a point $\left(x_{j}\right)_{(l)}$ and has metric $g_{i k(l)}$. One can introduce near $x_{j} a \cdot$ space of frame vectors $y_{i(l)}=g_{i k} e_{(l)}^{k}$ on the local chart $g_{i k}\left(x_{j}\right)$. Here a vector $e_{(l)}^{k}$ is carried paral lely itself from a point $x_{j}(\ell)$ to a point $x_{j}$. We determine the metric of $\mathrm{N}$-measured space $\mathrm{H}$ as $\mathrm{G}_{\ell n}=\mathrm{y}_{i(l)} \mathrm{y}_{(\mathrm{n})}^{\mathrm{i}}$, where the indices $n, l$ denote summation from 1 to $N$.

Are the isotropic geodesics of space $G_{\ell n}$ unstable?

Let $\mathbf{p}=\mathrm{y}_{(\ell)}^{i} \mathrm{P}_{\mathbf{i}}$ be a null vector, $\mathbf{P}_{\mathbf{i}} \mathbf{p}^{\mathbf{i}}=0$ and $\mathrm{P}_{\ell} \mathrm{p}^{\ell}=0$.

Humps deflect $p_{i}: P_{i} \rightarrow P_{i}+w_{i}$ and correspondingly $P_{\ell} \rightarrow p_{\ell}+w_{\ell}$, where $W_{\ell}=y_{(\ell)}^{i} w_{i}$. We must take into account the equations

$$
G_{\ell n} P^{\ell} \eta^{n}=0 \text {, }
$$




$$
G_{\ell n}\left(P^{l}+w^{l}\right)\left(P^{n}+w^{n}\right)=0 .
$$

The components of a deviation $W^{l}$ satisf $y$ the equations

$$
\frac{D^{2} W_{l}}{d \xi^{2}}=R_{l m n s} p^{m} p^{n} w^{s},
$$

where $R_{\ell m n s}$ is the Riemannian tensor of a space $G_{\ell n}, \xi$ is an affine parameter of an isotropic geodesic with a tangent vector $\mathrm{p}^{l}$ on a chart $\mathrm{g}_{\mathrm{ik}}$.

We consider the Eq. (1) on a surface $\left(\mathrm{y}_{(1)}, \mathrm{y}_{(2)}\right)$. Let $\mathrm{P}^{\ell}=\left(\mathrm{P}^{1}, \mathrm{p}^{2}, 0, \ldots, 0\right), \mathrm{w}^{\ell}=\left(w^{1}, w^{2}, \ldots, w^{N}\right)$ and we have

$$
w^{\ell}=G^{l n_{w}} w_{n}=y_{i}^{(l)} y^{i(n)} W_{n}
$$

Generally saying the vectors $y_{i}^{(l)}$ and $y_{i}^{(n)}$ are not parallel, so we may write

$$
y_{i}^{(l)} y^{i(n)}=\left(\delta^{l n}+\frac{n^{l} \eta^{n}}{\sigma}\right) \sigma .
$$

where $\delta^{\ell n}$ is the Kroneker symbol and vector $n^{\ell}$ characterises the difference of directions of vectors $y^{(l)}$ and $y^{(n)}, \sigma$ is proportional to some el ementary square. The equation (1) may be written as$$
\frac{D^{2} w_{l}}{d \xi^{2}}=-\left\{R_{1212} p^{2} \sigma\left(\delta^{1 n_{p^{2}}}-\delta^{2 n_{p^{1}}}\right)+R_{1212^{2}} \mathrm{p}^{2}\left(n^{1} \mathrm{p}^{2}-n^{2} \mathrm{p}^{1}\right) n^{n_{1}}\right\} W_{n}
$$

Now we see the deviation $W_{\ell}$ is changing if the space $\left\{y^{(\ell)}\right\}$ is anisotropic. The right hand of Eq. (2) is equal to zero if the space $H$ will be isotropic and $W_{l}=$ const. The deviation $W_{l}$ depends on the affine parameter $\xi$. The bracket in the right hand of Eq. (2) is the curvature of a surface $\left\{_{(1)}, y_{(2)}\right\}^{\prime}$ So the curvature is not positive, the isotropic geodesics are divergent. In this case we have a Theorem 1, which is formulate in the preface.

The angle $\psi$ between $\mathrm{P}^{\ell}$ and $\mathrm{P}^{\ell}+\mathrm{w}^{\ell}$ on the surface $\left\{y_{(2)}, y_{(3)}\right\}$ may be obtained from: 


$$
\cos \psi=\frac{\mathrm{P}_{\ell}\left(\mathrm{P}^{\ell}+\mathrm{w}^{\ell}\right)}{\left(\mathrm{P}_{\ell} \mathrm{P}^{\ell}\left(\mathrm{P}_{\mathrm{n}}+\mathrm{w}_{\mathrm{n}}\right)\left(\mathrm{P}^{\mathrm{n}}+\mathrm{w}^{\mathrm{n}}\right)\right)^{1 / 2}}
$$

where $\ell, n \geq 2$. One has $\cos \psi \rightarrow 1$ if the deviation $W_{\ell}$ is increasing.

From the standpoint of the modern ergodic theory such system (2) is mixing on a manifold $\{\psi\}$ Indeed, let $M_{0}$ be a possible manifold of initial angles $\psi_{0}: M_{0}=\int_{0}^{2 \pi} d \psi_{0}$. The dynamical system (2) has a sulmanifold $M=\int_{0}^{2 \pi} \cos \psi d \psi_{0}$. One can see $M \rightarrow M_{0}$ with an increasing $W_{f}$. The $|\cos \psi|$ is characteristic function of the system (2f).

2. Our Universe has probably a space-time with a humpy metric. We note al wa the time is closely bound up with a change of parameters of some physical system. So an arrow of time is defined along the direction of the change. One can distinguish a number of different arrows: the direction of time in which the entropy increases, the direction of time in which the Universe is expanding; the fact that the channels of $\mathrm{K}^{\circ}$ meson decays are a symmetric, the fact that we remember events in the past but not in the future (the psychological arrow time).

The phenomena have one common trait: "an observer" receives a new information as a phenomenon develops. An increase of the entropy in any point of a thermodynamical system corresponds to the fact that all information on boundary conditions ( $t$ he walls of a thermostat consist of a lot of particles) is "received" in a point by means of motion of particles of a system.

The expansion of the Universe is a fact that a new information of distant objects is received at any point when the object go into a horizon of an observer.

$\mathrm{K}^{\circ}$-meson decays occur with different probabilities. $R$. Penrose has suggested a hypothesis on a relation between the asymmetry and the temporal a symmetry. The $K^{0}$-a symmetry slightly perturbs all electrodynamic processes. Then an inf ormation on the $\mathrm{K}^{\circ}$-decay is received at any point of the $\mathrm{sp-}$ ace of electromagnetic degrees of freedom. The psychological time is related to new events too.

Therefore all times are the informative processes.

In accordance with the theory of General Relativity any physical phenomenon can be related to a space-time mani- 
fold with a dominant energy metric $\left(R_{i k} p^{i} p^{k} \geq 0\right)$. Then a humpy manifold is related to an ensemble of phenomena. The different humpy metric can describe all boundary conditions of thermodynamic systems, the surprising variety of the Universe and an inhomogeneous space of electromagnetic degrees of freedom. Probably one can find a relation between a humpy manifold and an inhomogeneous psyhological time. The use of a humpy manifold is adequate to the Nature if all space $H$ can have one arrow of time and the hemp of the time flow on different humps can be different.

Let us consider the propagation of the interactional signals in a humpy Universe. The isotropic geodesics are instable and $\nu=|\cos \phi|$ is a probability of a receiving of a signal in the direction $\psi$. If $\nu+1$ the signal may be received in any direction with equal probabilities. It is easy to convince that a mixing occurs on surfaces $\left\{y_{(1)}, y_{(l \geq 2} \geq\right\}$, where $\mathrm{y}_{(1)}$ is a coordinate time. Therefore the hypervector $\mathrm{p}=\left(\mathrm{P}^{1}, \mathrm{P}^{2}, \ldots, \mathrm{P}^{\mathrm{N}}\right)$ will be distributed over manifold of all directions $y_{(\ell>2)}$ with equal probabilities, and the corresponding direction cosines and $\left(\mathrm{P}^{\ell} /\left(\mathrm{P}^{\ell} \mathrm{P}_{\ell}\right)^{1 / 2}\right)_{\ell>2} \sim \frac{1}{\sqrt{N}}$. For $\mathrm{N} \gg 1$ the hypervector $\mathrm{P}^{\ell}$ will be almost perpendicular to all directions $y_{(\ell \geq 2)}$. Such hypervector can be in any point $\mathbf{x}_{\mathbf{j}}$

The degree of the uncertainty of receiving a signal or the Kolmogorov-Sinai entropy is $s=\frac{1}{\bar{N}} \sum_{\ell=2}^{N}\left|\cos \psi_{\ell}\right| \ell_{n}\left(1-\left|\cos \psi_{\ell}\right|\right)$. One can see $S$ increases with increasing $W_{\ell}$. The variable $S$ may be used as an informative time, and its arrow is related with a mixing of signals. 